VIII.

\title{
Bemerkungen zu der Differentialdiagnose der psychogenen Reaktionen mit besonderer Beriicksichtigung der im Kriege beobachteten psychischen Störungen.
}

\author{
Von \\ E. Meyer-Königsberg i. Pr.
}

Im letzten Heft des Archivs hat Raecke in einem Aufsatz: "Ueber hysterische und katatonische Situationspsychosen" mit Recht die Forderung erhoben, dass man bei diesen Formen psychischer Störung wieder mehr bestrebt sein solle, durch Vertiefung unserer Kenntnisse von der Symptomatologie des Zustandsbildes weiterzukommen. Er führt u. a. aus, wie unzulänglich die Anhaltspunkte, die der Nachweis und die besondere Berücksichtigung der Situation ergeben, für die Abgrenzung derartiger Zustandsbilder sind. Wie richtig diese Warnung vor Ueberschätzung der Situation für die Differentialdiagnose ist, ein wie unzureichender Notbehelf es ist, aus dem Gegebensein einer aussergewöhnlichen seelischen Erschütterung diagnostische Schlüsse zu ziehen, das lehren uns besonders die Beobachtungen im gegenwärtigen Kriege Eine schier unendliche Fülle unzweifelhaft psychogener Störungen trat uns entgegen, daneben ebenso zweifelsfreie Fälle von Dementia praecox, dann aber eine immer mehr zunehmende Zahl von Krankheitsbildern, die als psychogen erst ohne Weiterers imponierten, um dann immer mehr Züge der Dementia praecox aufzuweisen und anderseits mindestens ebenso zahlreich anscheinend einwandsfreie Dementia praecox-Fälle, die weiterhin als psychogene Störungen sich entpuppten, und schliesslich nicht wenige Beobachtungen, bei denen eine sichere Entscheidung; so lange der Zustand der gleiche blieb, unmöglich erschien.

Raecke spricht von einer "hysterischen" und einer "katatonischen Situationspsychose". Er gibt damit dem Gedanken Raum, dass in den gew öhnlichen Verlauf sonst durchaus verschiedoner Krankbeitsarten längere oder kürzere Zustandsbilder sich einschieben können, die in ibrer Entstehung an eine Situation, ein mit starkem Affekt einhergehendes Erlebnis, gebunden sind. 
Die Annahme wầre möglich, dass derartige psychotische Zustände, je mehr sie tatsächlich psychogene pathologisehe Reaktionen sind, in sich wesensgleich wären, einerlei welche Grundkrankheit den Boden für ibre Eutwicklung abgibt, und dass sie nur verschiedene Typen aufwiesen wie etwa die verschiedenen Reaktionsformen der symptomatischen Psychosen bei körperlichen Erkrankungen. Eine Unterscheidung müsste aber an und für sich möglich oder wenigstens denkbar sein, weil doch die Eigenheiten der Grundkrankheit bei genauer Beobachtung gleichsam durchschimmern werden, insofern jedenfalls als sie der psychogenen Reaktion eine besondere Färbung verleihen. Anderseits werden die psychotischen Erscheinungen, je reiner sie sind, um so mehr sich von der Eigenart der Grundlage freimachen. Bei derartigen Erwägungen ist aber auch immer zu bedenken, dass es sehr zweifelhaft ist, $o b$ und wie weit eine, jedenfalls voll entwickelte, Dementia praecox noch psychogene Reaktionsfähigkeit besitzt.

Die Hauptschwierigkeit wird, einerlei, wie wir uns zu den eben berührten Fragen stellen, immer darin liegen, dass die Dementia praecox vielfach Bilder hervorruft, die ohne oder jedenfalls ohne nachweisbar mit seelischen Erschätterungen im Zusammenhang zu stehen, äusserlich psychogenen Reaktionen mindestens sehr ähneln.

Es ergibt sich auch aus diesen Betrachtungen, dass die Differen tialdiagnose der psychogenen Reaktion') der Psychopathen gegenüber ähnlichen Bildern der Dementia praecox aus dem Nachweis eines stark affektbetonten Erlebnisses, einer Situation, nicht angängig ist, sondern dass sie in den Krankheitsbildern selbst fussen muss.

In Friedenszeiten sind die Schwierigkeiten hier auf bestimmte Gebiete, vor allem das der sogenannten Haftpsychosen beschränkt, wie uns ohne weiteres die Literatur hierüber erkennen lässt. Anders liegen die Dinge im Kriege. Schon bei der Zivilbevölkerung, besonders in den Grenzgebieten, aber auch sonst, spielen psychische Momente sehr vielfach mit - Angst vor dem Einfall feindlicher Truppen, vor Fliegern, Flucht vor dem Feinde, Drangsale während der feindlichen Okkupation, Verlust naher Angehöriger, Vermögensverfall $\mathfrak{u}$. dergl. werden mit mehr oder weniger Recht für die Entstehung geistiger Störungen angeschuldigt.

Ganz unbegrenzt ist aber bei den Kriegsteilnehmern die

1) Diese Namen möchte ich vorläufig für derartige Zustandsbilder, wie sie der hysterische Stupor und der Gansersche Dämmerzustand als bekannteste Typen darstellen, vorschlagen. 
Möglichkeit psychischer Schädigungen bei der Einstellung, Ausbildung, wie ganz besonders im Felde, deren Bewertung naturgemäss eine individuelle ist, von denen aber zumeist unbestreitbar ist, dass sie an und für sich imstande sind, das labile geistige Gleichgewicht Psychopathischveranlagter ernstlich zu erschüttern: Die völlige und plötzliche Loslösung aus den gewohnten Verhältnissen und der Familie, die Sorge um letztere, die Einordnung in die ganz neue Umgebung mit der ungewohnten Disziplin und den Anstrengungen des Dienstes, Granatexplosionen in nächster Nähe, tagelanges Aushalten im Granatfeuer, Ueberfälle und alle die plötzlichen Situationen des Krieges, weiter oft tagelange Anspannung aller Kräfte sind nur einzelne Beispiele; auch drohende Haft und Bestrafung fehlen nicht, aber neben diesen muss immer mit den anderen Momenten noch gerechnet werden. Und nun kommen, wie eingangs erwähnt, unausgesetzt Fälle mit stuporösen und verwandten Zuständen, Bilder mit Verworrenheit, Perseveration, monotonen Reden und Bewegungen u. ä. Erscheinungen zur Beobachtung, wo überall auslösende Momente psychischer Art sehr möglich oder direkt nachweisbar sind, wie soll uns da das stark affektbetonte seelische Erlebnis, die Situation, zur Diagnose verhelfen, die doch gerade jetzt, u. a. für die Frage der weiteren Dienstfähigkeit, so ungemein wichtig ist, da mit dem Vorliegen einer Dementia praecox Dienstfähigkeit absolut ausgeschlossen wäre.

Bei einer späteren Gelegenheit werde ich die einzelnen Beobachtungen mit dem Versuch der Unterscheidung mitteilen. Heute veranlasst mich die Bedeutung des Gegenstandes schon allgemein darauf hinzuweisen, dass, falls überhaupt etwas, nur das klinische Bild Aufschluss über die Grundkrankheit za geben vermag, wenn wir auch vielleicht von serologischen Untersuchungen (Abderhalden) und solchen des Blutes und elektrischer Art Unterstützung erwarten können. 